\title{
The Associated Classical Orthogonal Polynomials
}

\author{
Mizan Rahman * \\ School of Mathematics \& Statistics, Carleton University \\ Ottawa, ON K1S 5B6
}

\begin{abstract}
The associated orthogonal polynomials $\left\{p_{n}(x ; c)\right\}$ are defined by the 3 -term recurrence relation with coefficients $A_{n}, B_{n}, C_{n}$ for $\left\{p_{n}(x)\right\}$ with $c=0$, replaced by $A_{n+c}, B_{n+c}$ and $C_{n+c}, c$ being the association parameter. Starting with examples where such polynomials occur in a natural way some of the well-known theories of how to determine their measures of orthogonality are discussed. The highest level of the family of classical orthogonal polynomials, namely, the associated Askey-Wilson polynomials which were studied at length by Ismail and Rahman in 1991 is reviewed with special reference to various connected results that exist in the literature.
\end{abstract}

AMS subject classification: Primary 33A65; secondary 42C05

Key words and phrases: Classical orthogonal polynomials; Associated orthogonal polynomials; Associated Legendre, Laguerre, Hermite, Jacobi, q-ultraspherical, q-Jacobi and Askey-Wilson polynomials; Continued fractions, Stieltjes transform, Perron-Stieltjes inversion formula; Hypergeometric and basic hypergeometric series.

*Supported in part by NSERC grant \#A6197 


\section{Introduction}

For problems in heat conduction or potential theory that have axial symmetry the Laplace equation in cylindrical coordinates is

$$
\frac{1}{r} \frac{\partial}{\partial r}\left(r \frac{\partial V}{\partial r}\right)+\frac{\partial^{2} V}{\partial z^{2}}=0
$$

Discrete analogues of Laplace equation were considered by Courant, Friedricks and Lewy [11]. Boyer [7] studied the solutions of the following discretization of eqn. (1.1):

$$
\begin{aligned}
(m+ & \left.\frac{1}{2}\right) k\left(\frac{V(m h+h, n k)-V(m h, n k)}{h}\right) \\
& -\left(m-\frac{1}{2}\right) k\left(\frac{V(m h, n k)-V(m h-h, n k)}{h}\right) \\
& +m h\left(\frac{V(m h, n k+k)-2 V(m h, n k)+V(m h, n k-k)}{k}\right)=0,
\end{aligned}
$$

$m, n=1,2, \ldots,(h, k)$ represents the mesh-size, and the "boundary" condition is

$$
\frac{V(h, n k)-V(0, n k)}{h}+\frac{h}{4}\left(\frac{V(0, n k+k)-2 V(0, n k)+V(0, n k-k)}{k^{2}}\right)=0 .
$$

It is well-known that by separating the $r$ and $z$ variables in (1.1) one gets the Bessel function for the $r$-equation. Analogously, Boyer [7] sought solutions of (1.2) in the form

$$
V(m h, n k)=\phi_{m} e^{n \xi}
$$

where $\xi$ is related to the separation constant $\lambda$ by

$$
\lambda=\left(\frac{h}{k} \sinh \frac{\xi}{2}\right)^{2}
$$

Assuming that $\phi_{0}=1$ this leads to the 3 -term recurrence relation

$$
(2 m+1) \phi_{m+1}+(2 m-1) \phi_{m-1}-4 m(1-2 \lambda) \phi_{m}=0
$$

with

$$
\phi_{0}=1, \quad \phi_{1}=1-\lambda .
$$

Changing the variable by $\lambda=\frac{1-x}{2},(1.6)$ and (1.7) become

$$
\begin{gathered}
\left(n+\frac{1}{2}\right) p_{n+1}(x)=2 n x p_{n}(x)-\left(n-\frac{1}{2}\right) p_{n-1}(x), \quad n \geq 1, \\
p_{0}(x)=1, \quad p_{1}(x)=\frac{1+x}{2}, \quad \phi_{n}(\lambda)=p_{n}\left(\frac{1-x}{2}\right) .
\end{gathered}
$$


Boyer calls any solution of (1.8) a discrete Bessel function. However, it is clear that (1.8) is the $\nu=-\frac{1}{2}$ special case of the 3 - term recurrence relation of the so-called associated Legendre polynomials:

$$
(n+\nu+1) P_{n+1}(\nu, x)=(2 n+2 \nu+1) x P_{n}(\nu, x)-(n+\nu) P_{n-1}(\nu, x),
$$

see also Chihara [9].

In general, the 3-term recurrence relation satisfied by an orthogonal polynomial system $\left\{p_{n}(x)\right\}$ (OPS) is of the form

$$
p_{n+1}(x)=\left(A_{n} x+B_{n}\right) p_{n}(x)-C_{n} P_{n-1}(x), \quad n=0,1, \ldots,
$$

with $p_{-1}(x)=0, p_{0}(x)=1$. If $A_{n} A_{n+1} C_{n+1}>0, n=0,1, \ldots$, then there exists a positive measure $d \mu(x)$ with finite moments such that the following orthogonality relation

$$
\int_{-\infty}^{\infty} p_{n}(x) p_{m}(x) d \mu(x)=\lambda_{n} \delta_{m, n}, \quad m, n=0,1, \ldots, \lambda=A_{0}^{-1},
$$

holds.

Favard's theorem [9] assures us that the converse is also true. Finding the measure and the explicit solutions of (1.11) are a formidable task except in some special cases including the very important class of what is called the classical orthogonal polynomials. For instance, the Jacobi polynomials, $P_{n}^{(\alpha, \beta)}(x)$, the ultraspherical polynomial $C_{n}^{\lambda}(x)$, the Laguerre polynomial, $L_{n}^{\alpha}(x)$, and the Hermite polynomials, $H_{n}(x)$, satisfy, respectively, the recurrence relations

$$
\begin{aligned}
P_{n+1}^{(\alpha, \beta)}(x)= & \frac{(2 n+\alpha+\beta+1)(2 n+\alpha+\beta+2)}{2(n+1)(n+\alpha+\beta+1)} x \\
& \left.+\frac{\left(\alpha^{2}-\beta^{2}\right)(2 n+\alpha+\beta+1)}{2(n+1)(n+\alpha+\beta+1)(2 n+\alpha+\beta)}\right\} P_{n}^{(\alpha, \beta)}(x) \\
& -\frac{(n+\alpha)(n+\beta)(2 n+\alpha+\beta+2)}{(n+1)(n+\alpha+\beta+1)(2 n+\alpha+\beta)} P_{n-1}^{(\alpha, \beta)}(x), \\
C_{n+1}^{\lambda}(x) & =\frac{2(n+\lambda)}{n+1} x C_{n}^{\lambda}(x)-\frac{n+2 \lambda-1}{n+1} C_{n-1}^{\lambda}(x), \\
L_{n+1}^{\alpha}(x) & =\frac{2 n+\alpha+1-x}{n+1} L_{n}^{\alpha}(x)-\frac{n+\alpha}{n+1} L_{n-1}^{\alpha}(x), \\
H_{n+1}(x) & =2 x H_{n}(x)-2 n H_{n-1}(x),
\end{aligned}
$$

$$
\begin{aligned}
P_{n}^{(\alpha, \beta)}(x) & =\frac{(\alpha+1)_{n}}{n !}{ }_{2} F_{1}(-n, n+\alpha+\beta+1 ; \alpha+1 ;(1-x) / 2), \\
C_{n}^{\lambda}(x) & =\sum_{k=0}^{n} \frac{(\lambda)_{k}(\lambda)_{n-k}}{k !(n-k) !} \cos (n-2 k) \theta \\
& =\frac{(2 \lambda)_{n}}{n !}{ }_{2} F_{1}\left(-n, n+2 \lambda ; \lambda+\frac{1}{2} ;(1-x) / 2\right), \quad \cos \theta=x
\end{aligned}
$$




$$
\begin{aligned}
L_{n}^{\alpha}(x) & =\frac{(\alpha+1)_{n}}{n !}{ }_{1} F_{1}(-n ; \alpha+1 ; x), \\
H_{n}(x) & =n ! \sum_{k=0}^{\left[\frac{n}{2}\right]} \frac{(-1)^{k}(2 x)^{n-2 k}}{k !(n-2 k) !} .
\end{aligned}
$$

Discrete classical OPS that include the Krawtchouk, Meixner and Charlier polynomials, see [13], have also similar simple expressions in terms of hypergeometric functions. Usually the measures of orthogonality of the classical OPS can be determined in a fairly straightforward manner, often by quite elementary methods. However, things can change quite dramatically even on an apparently harmless and innocent-looking alteration of the 3-term recurrence relation:

$$
p_{n+1}(x)=\left(A_{n+c} x+B_{n+c}\right) p_{n}(x)-C_{n+c} p_{n-1}(x), \quad n=0,1, \ldots,
$$

with $p_{-1}(x)=0, p_{0}(x)=1, A_{n+c} A_{n+c+1} C_{n+c+1}>0, n=0,1, \ldots$, where $c$ is an additional parameter such as $c=-1 / 2$ that was used in (1.8). Polynomial solutions of (1.21) satisfying the given initial conditions are called the associated orthogonal polynomials (i.e. associated with the ones with $c=0$ ).

Apart from their possible use in physical models, exemplified by the discretized Laplace eqn. (1.2), they occur in a natural way, for positive integer values of $c$, in the theory of the solutions of the original system satisfying (1.11).

It is well-known that closely related to (1.11) is the continued fraction

$$
F(x)=\frac{1}{\mid A_{0} x+B_{0}}-\frac{C_{1} \mid}{\mid A_{1} x+B_{1}}-\frac{C_{2} \mid}{\mid A_{2} x+B_{2}}-\ldots
$$

see, for example, [9] and [2]. The $n$th convergent of this continued fraction, say, $P_{n}^{(1)}(x) / P_{n}(x)$, is a rational function where the denominator polynomial $P_{n}(x)$ satisfies (1.11) with the same initial conditions, but the numerator polynomial $P_{n}^{(1)}(x)$ satisfy the same recurrence relation, but a different initial condition, namely,

$$
P_{0}^{(1)}(x)=0, \quad P_{1}^{(1)}(x)=1 .
$$

Hence $P_{n}^{(1)}(x)$ is a polynomial of precise degree $n-1$, and so it satisfies the associated equation

$$
r_{n+1}(x)=\left(A_{n+1} x+B_{n+1}\right) r_{n}(x)-C_{n+1} r_{n-1}(x),
$$

with $r_{-1}(x)=0, r_{0}(x)=1, r_{n-1}(x)=P_{n}^{(1)}(x)$. In this manner one can generate a whole sequence of polynomial systems $P_{n}^{(k)}(x)$, the so-called numerator polynomials, [9], satisfying the recurrence relation

$$
r_{n+1}(x)=\left(A_{n+k} x+B_{n+k}\right) r_{n}(x)-C_{n+k} r_{n-1}(x),
$$

with $r_{-1}(x)=0, r_{0}(x)=1, k=1,2, \ldots$ 
Whether or not the continued fraction in (1.22) converges it is clear that $P_{n}^{(1)}(x) / P_{n}(x)$ provides an $[n-1 / n]$ Padé approximant to $F(x)$. However, when the continued fraction does converge, we write

$$
F(x)=\lim _{n \rightarrow \infty} \frac{P_{n}^{(1)}(x)}{P_{n}(x)} .
$$

Wimp [50] considered the $[n / n]$ Padé approximant, $A_{n}(z) / B_{n}(z)$, for the logarithmic derivative of the confluent hypergeometric function ${ }_{1} F_{1}(a ; b ; z)$ and used the polynomials $A_{n}(z)$ and $B_{n}(z)$ to derive a discrete orthogonality relation for the Lommel and the corresponding associated polynomials.

The associated orthogonal polynomials also appear in a natural way in the birth-anddeath processes, studied by Karlin and McGregor [28], [29], see also Ismail, Letessier and Valent [22]. Let

$$
p_{m n}(t)=\operatorname{Prob}\{X(t)=n \mid X(0)=m\},
$$

the transition probabilities of a birth-and-death process, be given by

$$
p_{m n}(t)= \begin{cases}\lambda_{m} t+o(t), & n=m+1 \\ \mu_{m} t+o(t), & n=m-1 \\ 1-\left(\lambda_{m}+\mu_{m}\right) t+o(t), & n=m,\end{cases}
$$

where $\lambda_{n}$ and $\mu_{n}$ are the birth and death rates at the state $n$, respectively. With $\lambda_{n}>0$, $\mu_{n+1}>0, n \geq 0, \mu_{0} \geq 0$, Karlin and McGregor [28] proved that

$$
p_{m n}(t)=\pi_{n} \int_{0}^{\infty} e^{-x t} Q_{m}(x) Q_{n}(x) d \mu(x),
$$

where $\pi_{0}=1, \pi_{n}=\left(\lambda_{0} \lambda_{1} \ldots \lambda_{n-1}\right) /\left(\mu_{1} \mu_{2} \ldots \mu_{n}\right), n>0$, and $\left\{Q_{n}(x)\right\}$ are polynomials orthogonal w.r.t. $d \mu$. If we set $F_{n}(x)=\pi_{n} Q_{n}(x)$, then the orthogonality relation for $\left\{F_{n}(x)\right\}$ is

$$
\int_{0}^{\infty} F_{n}(x) F_{m}(x) d \mu(x)=\pi_{n} \delta_{m n}
$$

where the polynomials $F_{n}(x)$ satisfy the 3 -term recurrence relation

$$
F_{n+1}(x)=\frac{\lambda_{n}+\mu_{n}-x}{\mu_{n+1}} F_{n}(x)-\frac{\lambda_{n-1}}{\mu_{n+1}} F_{n-1}(x),
$$

with

$$
F_{0}(x)=1, \quad F_{1}(x)=\left(\lambda_{0}+\mu_{0}-x\right) / \mu_{1} .
$$

For linear birth-and-death models Ismail, Letessier and Valent [22] use the two cases: (i) $\lambda_{n}=n+\alpha+c+1, \mu_{n}=n+c, n \geq 0, c>0$; (ii) $\lambda_{n}=n+\alpha+c+1, n \geq 0 ; \mu_{0}=0$, $\mu_{n}=n+c, n \geq 1$. In case (i) the recurrence relation (1.31) can be rewritten in the form

$$
L_{n+1}^{\alpha}(x ; c)=\frac{2 n+2 c+\alpha+1-x}{n+c+1} L_{n}^{\alpha}(x ; c)-\frac{n+\alpha+c}{n+c+1} L_{n-1}^{\alpha}(x ; c),
$$


which is the relation associated to (1.15) with $n$ replaced by $n+c$ in the coefficients. Askey and Wimp [5] found the measure of orthogonality for these associated Laguerre polynomials $L_{n}^{\alpha}(x ; c)$, as well as their explicit polynomial form:

$$
L_{n}^{\alpha}(x ; c)=\frac{(\alpha+1)_{n}}{n !} \sum_{k=0}^{n} \frac{(-n)_{k} x^{k}}{(c+1)_{k}(\alpha+1)_{k}}{ }_{3} F_{2}\left[\begin{array}{c}
k-n, k+1-\alpha, c \\
k+c+1,-\alpha-n
\end{array} ; 1\right] .
$$

By a different method the measures of orthogonality were found in [22], in addition to the explicit form of the second system of associated Laguerre polynomials that corresponds to case (ii):

$$
\mathcal{L}_{n}^{\alpha}(x ; c)=\frac{(\alpha+1)_{n}}{n !} \sum_{k=0}^{n} \frac{(-n)_{k} x^{k}}{(c+1)_{k}(\alpha+1)_{k}}{ }_{3} F_{2}\left[\begin{array}{c}
k-n, k-\alpha, c \\
k+c+1,-\alpha-n
\end{array} ; 1\right] .
$$

In a later paper the same authors [23] considered the polynomials associated with symmetric birth and death processes with quadratic rates: $\lambda_{n}=(n+a)(n+b), n \geq 0$, $\mu_{n}=(n+\alpha)(n+\beta), n>0, \mu_{0}=0$ or $\mu_{0}=\alpha \beta$. They obtained the absolutely continuous measure for the resulting associated continuous dual Hahn orthogonal polynomials as well as the following explicit formula:

$$
\begin{aligned}
P_{n}(x ; & a, b, \alpha, \beta, \eta) \\
= & \frac{(a)_{n}}{n !} \sum_{k=0}^{n} \frac{(-n)_{k}\left(a+\gamma+i \sqrt{x-\gamma^{2}}\right)_{k}\left(a+\gamma-i \sqrt{x-\gamma^{2}}\right)_{k}}{(a)_{k}(\alpha+1)_{k}(\beta+1)_{k}} \\
& \times \sum_{j=0}^{k} \frac{(\alpha)_{j}(\beta)_{j}(a+\eta-1)_{j}}{j !\left(a+\gamma+i \sqrt{x-\gamma^{2}}\right)_{j}\left(a+\gamma-i \sqrt{x-\gamma^{2}}\right)_{j}},
\end{aligned}
$$

where $\gamma=(1+\alpha+\beta-a-b) / 2$ and $\eta=0$ when $\mu_{0}=0$ and $\eta=1$ when $\mu_{0}=\alpha \beta \neq 0$. Note that $P_{n}(x ; a, b, \alpha, \beta, \eta)$ reduces to the continuous dual Hahn polynomials, see Andrews and Askey [1] and Askey and Wilson [3] when $\alpha=0$ or $\beta=0$.

It may be hazardous to speculate who was the first one to study the associated orthogonal polynomials or who used the adjective "associated" to describe them, but Humbert's 1918 paper [20] is the earliest work that I could find in the literature. Hahn [19] seems to be the first to study the associated Laguerre polynomials. Although he did not find their orthogonality relation he found the fourth order differential equation that they satisfy by first expressing them as sums of products of confluent hypergeometric functions. Of all the earlier works on associated orthogonal polynomials, however, Pollaczek's work [38-41] was probably the most important because his results constituted a significant departure from the classical orthogonal polynomials (belonging to the Szegö class) on one hand, and a generalization to the associated ones on the other. In its full generality Pollaczek [41] gave the following 3 -term recurrence relation

$$
(n+c+1) P_{n+1}^{\lambda}(x)=2[(n+c+\lambda+a) x+b] P_{n}^{\lambda}(x)-(n+c+2 \lambda-1) P_{n-1}^{\lambda}(x),
$$

with $P_{-1}^{\lambda}(x)=0, P_{0}^{\lambda}(x)=1,|x| \leq 1$, and either $a>|b|, 2 \lambda+c>0, c \geq 0$ or $a>|b|$, $2 \lambda+c \geq 1, c>-1$. Pollaczek proved the orthogonality of $\left\{P_{n}^{\lambda}(x)\right\}$ on $[-1,1]$ wrt the weight 
function

$$
\begin{aligned}
w^{(\lambda)}(x ; a, b, c)= & \frac{(2 \sin \theta)^{2 \lambda-1} e^{(2 \theta-\pi) t}}{2 \pi \Gamma(2 \lambda+c) \Gamma(c+1)} \\
& \times|\Gamma(\lambda+c+i t)|^{2}\left|{ }_{2} F_{1}\left(1-\lambda+i t, c ; c+\lambda+i t ; e^{2 i \theta}\right)\right|^{-2}
\end{aligned}
$$

where $t=(a \cos \theta+b) / \sin \theta, x=\cos \theta$, and derived the following expression:

$$
\begin{aligned}
P_{n}^{\lambda}(x) & =P_{n}^{\lambda}(x ; a, b, c) \\
& =\frac{A_{-1} B_{n}-A_{n} B_{-1}}{A_{-1} B_{0}-B_{-1} A_{0}},
\end{aligned}
$$

with

$$
\begin{aligned}
A_{n}= & \frac{\Gamma(2 \lambda+c+n) e^{i(c+n) \theta}}{\Gamma(c+n+1) \Gamma(2 \lambda)}{ }_{2} F_{1}\left(-c-n, \lambda+i t ; 2 \lambda ; 1-e^{-2 i \theta}\right), \\
B_{n}= & \frac{\Gamma(1-\lambda+i t) \Gamma(1-\lambda-i t)}{\Gamma(2-2 \lambda)}(2 \sin \theta)^{1-2 \lambda} e^{i(2 \lambda+c+n-1) \theta} \\
& \times{ }_{2} F_{1}\left(1-2 \lambda-c-n, 1-\lambda+i t ; 2-2 \lambda ; 1-e^{-2 i \theta}\right),
\end{aligned}
$$

$n=-1,0,1, \ldots$, provided $2 \lambda$ is not an integer, see also [13].

Taking the limit $a \rightarrow 0$, replacing $\theta$ and $t$ by $\phi$ and $x$, respectively, one obtains the limiting relation:

$$
\begin{aligned}
& (n+c+1) P_{n+1}^{\lambda}(x) \\
& \quad=2[(n+c+\lambda) \cos \phi+x \sin \phi] P_{n}^{\lambda}(x)-(n+c+2 \lambda-1) P_{n-1}^{\lambda}(x),
\end{aligned}
$$

with $P_{-1}^{\lambda}(x)=0, P_{0}^{\lambda}(x)=1,0<\phi<\pi, 2 \lambda+c>0, c \geq 0$, or $0<\phi<\pi, 2 \lambda+c \geq 1$, $c>-1$. The resulting polynomials, called by Askey and Wimp [5] the associated MeixnerPollaczek polynomials, (Meixner [34] had also found them and their orthogonality relation when $c=0$ ) are orthogonal on the infinite interval $-\infty<x<\infty$ wrt the same weight function (1.38) with appropriate replacement of the variables. If we replace $\lambda$ and $x$ in (1.41) by $\frac{1}{2}(\alpha+1)$ and $-x / 2 \sin \phi$, respectively, and take the limit $\phi \rightarrow 0$, then (1.41) becomes the 3 -term recurrence relation for the associated Laguerre polynomials, $L_{n}^{\alpha}(x ; c)$, as was observed by Pollaczek in [40]. Askey and Wimp [5] took advantage of his property to obtain the weight function for the orthogonality of $\left\{L_{n}^{\alpha}(x ; c)\right\}$ on $0<x<\infty$, namely,

$$
W^{\alpha}(x ; c)=\frac{x^{\alpha} e^{-x}}{\left|\Psi\left(c, 1-\alpha ; x e^{-\pi i}\right)\right|^{2}},
$$

where

$$
\Psi(a, b ; x)=\frac{1}{\Gamma(a)} \int_{0}^{\infty} e^{-x t} t^{a-1}(1+t)^{c-a-1} d t, \quad \operatorname{Re} a>0
$$

is the second solution of the confluent hypergeometric equation. Askey and Wimp [5] also found the weight function for the associated Hermite polynomials $H_{n}(x ; c)$ :

$$
w(x ; c)=\left|D_{-c}(i x \sqrt{2})\right|^{-2},
$$


where $\lim _{b \rightarrow \infty} b^{a / 2} \Psi(a, b+1 ; x \sqrt{b}+b)=e^{x^{2} / 4} D_{-a}(x)$, and the expression

$$
H_{n}(x ; c)=\sum_{k=1}^{\left\lfloor\frac{n}{2}\right\rfloor} \frac{(-2)^{k}(c)_{k}(n-k) !}{k !(n-2 k) !} H_{n-2 k}(x) .
$$

These results were derived by a different method in [22].

In section 2 we shall give a brief summary of the methods of determining the spectral measures $d \mu(x)$ and in section 3 we discuss in some details the case of the two families of Askey-Wilson polynomials. Finally we consider some special and limiting cases in section 4 .

\section{Determination of the spectral measure $d \mu(x)$}

As we saw in the previous section the existence of a positive measure $d \mu(x)$ for an OPS $\left\{p_{n}(x)\right\}$ defined by the 3 -term recurrence relation (1.11) or (1.21) along with the stated conditions is guaranteed by Favard's theorem, but nothing else can be said about this measure without a good deal of additional work. First, one has to determine the bounds of the support of $d \mu$ for which one can use a characterization theorem of Wall and Wetzel [46] in terms of the so-called chain sequences to find the true interval of orthogonality, or an alternate approach suggested by Chihara [9], [10]. See [21] for a brief summary of Chihara's ideas. If the support turns out to be $(0, \infty)$ or $(-\infty, \infty)$ then the unboundedness of it poses the additional problem of whether or not one has a determined or an undetermined moment problem. For a comprehensive analysis of this problem see [44]. However, for a finite interval $[a, b]$ the measure is necessarily unique, see [45], and can be obtained, in principle, in 4 different ways.

There is another approach to the associated OPS problem, mainly due to Grunbaum [15], [16], who looks at them from the point of view of the bispectral problem. Space limitations, however, restrict us to just refer the reader to his papers.

I. Method of moments. The moments of the measure $d \mu(x)$ are defined by $\mu_{n}=\int_{a}^{b} x^{n} d \mu(x)$. Assuming that the measure is normalized to unity so that $\mu_{0}=1$, the first few moments can be computed by using the recurrence relation (1.11) and the orthogonality property (1.12), whether or not they are for the associated polynomials. A pattern may or may not emerge from these calculations. If it does then a guess can be made about the general formula and, hopefully, proved by induction. Then one can compute the function

$$
F(z)=\sum_{n=0}^{\infty} \mu_{n} z^{-n-1}, \quad|z|>r
$$

which is really an asymptotic expansion of $F(z)$ as $z \rightarrow \infty$, that converges in the exterior of any circle $|z|=r$ containing the interval of orthogonality in its interior. 
For $z \notin[a, b]$, it is well- known that

$$
F(z)=\int_{a}^{b} \frac{d \mu(t)}{z-t}
$$

is the Stieltjes transform of $d \mu(t)$. Then the familiar Perron- Stieltjes inversion formula tells us that

$$
\mu\left(t_{2}\right)-\mu\left(t_{1}\right)=\frac{1}{2 \pi i} \lim _{\varepsilon \rightarrow 0^{+}} \int_{t_{1}}^{t_{2}}\{F(t-i \varepsilon)-F(t+i \varepsilon)\} d t
$$

if and only if (2.2) holds, see [21], [44].

II. The generating function method. When the interval of orthogonality is bounded (or, more generally, when the moment problem is determined) one can show that

$$
F(z)=\lim _{n \rightarrow \infty} \frac{P_{n}^{(1)}(z)}{P_{n}(z)}=\int_{-\infty}^{\infty} \frac{d \mu(t)}{z-t}
$$

which may be compared with (1.26), where $z$ is in the complex plane cut along the support of $d \mu$, and the convergence is uniform on compact subsets of this cut plane, see $[44]$.

Note that it is not necessary to compute the denominator and numerator polynomials explicitly, only their asymptotic behaviour as $n \rightarrow \infty$. It was pointed out in [2] that the asymptotic method of Darboux [45] can be applied to the generating functions of both $\left\{P_{n}(x)\right\}$ and $\left\{P_{n}^{(1)}(x)\right\}$ to determine their asymptotic behaviour. Darboux's theorem, as stated in [36], is as follows.

Let

$$
f(z)=\sum_{n=-\infty}^{\infty} a_{n} z^{n}
$$

be the Laurent expansion of an analytic function $f(z)$ in an annulus $0<|z|<r<\infty$. By Cauchy's formula

$$
a_{n}=\frac{1}{2 \pi i} \int_{C} \frac{f(z)}{z^{n+1}} d z
$$

where $C$ is a simple closed contour in the annulus around $r=0$. Let $r$ be the distance from the origin of the nearest singularity of $f(z)$, and suppose we can find a "comparison" function $g(z)$ such that

(i) $g(z)$ is holomorphic in $0<|z|<r$;

(ii) $f(z)-g(z)$ is continuous in $0<|z|<r$ (this can be weakened, see [36]);

(iii) the coefficients $b_{n}$ in the Laurent expansion

$$
g(z)=\sum_{n=-\infty}^{\infty} b_{n} z^{n}
$$


have known asymptotic behaviour. Then

$$
a_{n}=b_{n}+o\left(r^{-n}\right), \quad n \rightarrow \infty .
$$

Since $G^{(k)}(z, t):=\sum_{n=0}^{\infty} P_{n}^{(k)}(z) t^{n}$, the generating function of the orthogonal polynomials $P_{n}^{(k)}(z)$, can be computed from their 3-term recurrence relations (at least in principle) without the explicit knowledge of the polynomials themselves, one can usually locate its singularities in the complex plane which enables one to construct simpler "comparison" functions with known asymptotic behaviour. Therefore, this method is inherently simpler to use than the other methods, and have been used in a number of works on associated OPS. See, for example, [2], [22], [23], [21], [8].

III. Special function methods. These methods are usually quite computational and rely more on inspired guesses than on the elaborate machinery of the theory of general OPS. The first step is to find an explicit form of the polynomial solution of the recurrence relation. In the case of classical orthogonal polynomials this solution also satisfies a second-order differential or difference equation which can be transformed, if necessary, into a self-adjoint form. The weight function usually reveals itself from that form. Consider, for example, the Askey-Wilson polynomials:

$$
\begin{aligned}
& P_{n}(x ; a, b, c, d \mid q) \\
& \quad={ }_{4} \phi_{3}\left[\begin{array}{c}
q^{-n}, a b c d q^{n-1}, a e^{i \theta}, a e^{-i \theta} \\
a b, a c, a d
\end{array} ; q, q\right], \quad n=0,1, \ldots,
\end{aligned}
$$

where the ${ }_{4} \phi_{3}$ series is a balanced and terminating basic hypergeometric series defined in [14], see also [3]. Askey and Wilson [4] had shown in an earlier paper that if one of $a, b, c, d$ is of the form $q^{-n}, n=0,1, \ldots$, then

$$
\phi(a, b):={ }_{4} \phi_{3}\left[\begin{array}{c}
a, b, c, d \\
e, \quad f, g
\end{array} ; q, q\right], \quad \text { efg }=a b c d q,
$$

satisfies the contiguous relation

$$
A \phi\left(a q^{-1}, b q\right)+B \phi(a, b)+C \phi\left(a q, b q^{-1}\right)=0,
$$

where

$$
\begin{aligned}
& A=b(1-b)(a q-b)(a-e)(a-f)(a-g), \\
& C=-a(1-a)(b q-a)(b-e)(b-f)(b-g), \\
& B=C-A+a b(a-b q)(a-b)(a q-b)(1-c)(1-d) .
\end{aligned}
$$

So, if we replace $a, b$ by $q^{-n}$ and $a b c d q^{n-1}$, respectively, and then $c, d, e, f, g$ by $a e^{i \theta}, a e^{-i \theta}, a b, a c, a d$, in that order, then we obtain the 3 -term recurrence relation for $p_{n}(x ; a, b, c, d \mid q)$ :

$$
2 x p_{n}(x)=A_{n} p_{n+1}(x)+B_{n} p_{n}(x)+C_{n} p_{n-1}(x),
$$


with

$$
\begin{aligned}
& A_{n}=\frac{a^{-1}\left(1-a b q^{n}\right)\left(1-a c q^{n}\right)\left(1-a d q^{n}\right)\left(1-a b c d q^{n-1}\right)}{\left(1-a b c d q^{2 n-1}\right)\left(1-a b c d q^{2 n}\right)} \\
& C_{n}=\frac{a\left(1-b c q^{n-1}\right)\left(1-b d q^{n-1}\right)\left(1-c d q^{n-1}\right)\left(1-q^{n}\right)}{\left(1-a b c d q^{2 n-2}\right)\left(1-a b c d q^{2 n-1}\right)} \\
& B_{n}=a+a^{-1}-A_{n}-C_{n} .
\end{aligned}
$$

However, if we had replaced $a, b, c, d, e, f, g$ by $a e^{i \theta}, a e^{-i \theta}, q^{-n}, a b c d q^{n-1}, a b, a c, a d$, respectively, with $x=\cos \theta$, then we would obtain the following relation from (2.11) and (2.12):

$$
\begin{aligned}
& q^{-n}\left(1-q^{n}\right)\left(1-a b c d q^{n-1}\right) r_{n}\left(e^{i \theta}\right) \\
& \quad=\lambda(-\theta)\left\{r_{n}\left(q^{-1} e^{i \theta}\right)-r_{n}\left(e^{i \theta}\right)\right\}+\lambda(\theta)\left\{r_{n}\left(q e^{i \theta}\right)-r_{n}\left(e^{i \theta}\right)\right\},
\end{aligned}
$$

where $r_{n}\left(e^{i \theta}\right):=p_{n}(\cos \theta ; a, b, c, d \mid q)$, and

$$
\lambda(\theta)=\frac{\left(1-a e^{i \theta}\right)\left(1-b e^{i \theta}\right)\left(1-c e^{i \theta}\right)\left(1-d e^{i \theta}\right)}{\left(1-e^{2 i \theta}\right)\left(1-q e^{2 i \theta}\right)} .
$$

Askey and Wilson [3] manipulated (2.15) to obtain the second-order divided-difference equation in a "self-adjoint" form:

$$
D_{q}\left[w\left(x ; a q^{1 / 2}, b q^{1 / 2}, c q^{1 / 2}, d q^{1 / 2}\right) D_{q} p_{n}(x)\right]+\lambda_{n} w(x ; a, b, c, d) p_{n}(x)=0
$$

where

$$
w(x ; a, b, c, d) d x=\frac{\left(e^{2 i \theta}, e^{-2 i \theta} ; q\right)_{\infty}}{\left|\left(a e^{i \theta}, b e^{i \theta}, c e^{i \theta}, d e^{i \theta} ; q\right)_{\infty}\right|^{2}} \frac{d x}{\sqrt{1-x^{2}}}
$$

is the absolutely continuous measure on $[-1,1]$.

Unfortunately, however, this simple device does not seem to work for nonclassical OPS, and certainly not for the associated ones, classical or otherwise. Magnus [30] showed that the OPS belonging to what he calls the Laguerre-Hahn class, defined in terms of a Riccati-type difference equation, that includes the classical OPS along with the associated ones, generally satisfy linear difference or differential equations of fourth order. Magnus did not attempt to obtain the measure of orthogonality for the associated Askey-Wilson polynomials, $p_{n}^{\alpha}(x ; a, b, c, d \mid q)$, which satisfy the 3 -term recurrence relation (2.13) with $A_{n}, B_{n}, C_{n}$ replaced by $A_{n+\alpha}, B_{n+\alpha}, C_{n+\alpha}$, but gave a scheme of how to derive the corresponding fourth order difference equation. Wimp [49], however, was able to find an explicit fourth order differential equation for the associated Jacobi polynomials which are the $q \rightarrow 1$ limit cases of $p_{n}^{\alpha}\left(x ; q^{1 / 2}, q^{\alpha+1 / 2},-q^{\beta+1 / 2},-q^{1 / 2} \mid q\right)$.

Considering the complexity of the weight function $w^{\lambda}(x ; a, b, c)$ in (1.37) for the Pollaczek polynomials it would appear that guessing the measure of orthogonality 
for the associated OPS or to derive it from special function formulas would be a daunting task indeed. Barrucand and Dickinson [6] discovered the weight function for the associated Legendre polynomials satisfying eqn. (1.10) by a clever manipulation of the Legendre functions of both kinds, without realizing that their results follow as special cases of Pollaczek's formulas given in (1.36)- -(1.39), see also [12], [38].

One may describe the special function methods and even the moment methods pretty adhoc and simple-minded, but they can be quite effective in determining the measure of orthogonality, sometimes even when it is not unique, for example, the $V_{n}(x ; a)$ polynomials of Al-Salam and Carlitz, see [9].

IV. Method of minimal solutions. This method, extensively used by Masson [31$33]$ and his collaborators [17-18], [21], [26], relies on the following ideas. A solution $X_{n}^{(s)}$ of the 3-term recurrence relation

$$
X_{n+1}=A_{n} X_{n}+B_{n} X_{n-1}, \quad n \geq 0,
$$

is a minimal (or subdominant) solution if for any other linearly independent solution $X_{n}^{(d)}$ (dominant) one has the property

$$
\lim _{n \rightarrow \infty} \frac{X_{n}^{(s)}}{X_{n}^{(d)}}=0 .
$$

Pincherle's theorem (see [31] and the references therein) states that (2.20) is a necessary and sufficient condition for the convergence of the continued fraction that corresponds to (2.19), namely,

$$
\frac{B_{0}}{A_{0}+} \frac{B_{1}}{A_{1}+} \frac{B_{2}}{A_{2}+} \cdots .
$$

In case of convergence the limit is simply given by $-\frac{X_{0}^{(s)}}{X_{-1}^{(s)}}$, assuming, of course, that $B_{n} \neq 0, n \geq 0$. When a minimal solution exists it is unique up to a multiple independent of $n$. However, it was noted in [33] by means of an example that the minimal solution may change in different parts of the complex plane or from $n \geq 0$ to $n \leq 0$. An example of how to construct a minimal solution, when it exists, by first deriving a set of solutions of (2.19) (any two of them being linearly independent) is given in [17].

\section{Associated Askey-Wilson polynomials}

The associated polynomials that generalize the Askey-Wilson polynomials given in (2.9) are solutions of the 3-term recurrence relation

$$
\left(z+z^{-1}-a-a^{-1}+A_{n+\alpha}+C_{n+\alpha}\right) p_{n}^{\alpha}(x)
$$




$$
=A_{n+\alpha} p_{n+1}^{\alpha}(x)+C_{n+\alpha} p_{n-1}^{\alpha}(x), \quad n=0,1,2, \ldots,
$$

with $p_{-1}^{\alpha}(x)=0, p_{0}^{\alpha}(x)=1 ; A_{n+\alpha}, C_{n+\alpha}$ being the same as in (2.14) with $n$ replaced by $n+\alpha$. It was shown in [27] that the two linearly independent solutions of (3.1) are

$$
\begin{aligned}
r_{n+\alpha}= & \frac{\left(a b q^{n+\alpha}, a c q^{n+\alpha}, a d q^{n+\alpha}, b c d q^{n+\alpha} / z ; q\right)_{\infty}}{\left(b c q^{n+\alpha}, b d q^{n+\alpha}, c d q^{n+\alpha}, a z q^{n+\alpha} ; q\right)_{\infty}}\left(\frac{a}{z}\right)^{n+\alpha} \\
& \times{ }_{8} W_{7}\left(b c d / q z ; b / z, c / z, d / z, a b c d q^{n+\alpha-1}, q^{-\alpha-n} ; q, q z / a\right)
\end{aligned}
$$

and

$$
\begin{aligned}
s_{\alpha+n}= & \frac{\left(a b c d q^{2 n+2 \alpha}, b z q^{\alpha+n+1}, c z q^{n+\alpha+1}, d z q^{n+\alpha+1}, b c d z q^{n+\alpha} ; q\right)_{\infty}}{\left(b c q^{n+\alpha}, b d q^{n+\alpha}, c d q^{n+\alpha}, q^{n+\alpha+1}, b c d z q^{2 n+2 \alpha+1} ; q\right)_{\infty}}(a z)^{n+\alpha} \\
& \times{ }_{8} W_{7}\left(b c d z q^{2 n+2 \alpha} ; b c q^{n+\alpha}, b d q^{n+\alpha}, c d q^{n+\alpha}, q^{n+\alpha+1}, z q / a ; q, a z\right),
\end{aligned}
$$

where

$$
\begin{aligned}
& { }_{2 r+1} W_{2 r}\left(a ; a_{1}, a_{2}, \ldots, a_{2 r-2} ; q, z\right) \\
& \quad:={ }_{2 r+1} \phi_{2 r}\left[\begin{array}{l}
\left.a, q a^{1 / 2},-q a^{1 / 2}, a_{1}, \ldots, a_{2 r-2} ; q, z\right] \\
a^{1 / 2},-a^{1 / 2}, q a / a_{1}, \ldots, q a / a_{2 r-2}
\end{array}\right]
\end{aligned}
$$

is a very-well-poised basic hypergeometric series, see [14] for definitions, properties and relevant formulas. Taking a linear combination of these solutions along with the given initial conditions $\left(p_{-1}^{\alpha}=0, p_{0}^{\alpha}=1\right)$ leads to the following expression:

$$
\begin{aligned}
p_{n}^{\alpha}(x)= & \frac{\left(b c, b d, c d, b c q^{\alpha-1}, b d q^{\alpha-1}, c d q^{\alpha-1}, q^{\alpha}, a z ; q\right)_{\infty} z a^{1-2 \alpha}}{\left(1-a b c d q^{2 \alpha-2}\right)\left(a b q^{\alpha}, a c q^{\alpha}, a d q^{\alpha}, a b c d q^{\alpha-1}, b z, c z, d z, b c d / z ; q\right)_{\infty}} \\
& \times\left\{s_{\alpha-1}(z) r_{n+\alpha}(z)-r_{\alpha-1}(z) s_{n+\alpha}(z)\right\},
\end{aligned}
$$

where $2 x=z+z^{-1}$, from which one gets the asymptotic formula:

$$
\lim _{n \rightarrow \infty}(z / a)^{n} p_{n}^{\alpha}(x)=\frac{(a z)^{1-\alpha}\left(a z, b c q^{\alpha-1}, b d q^{\alpha-1}, c d q^{\alpha-1}, q^{\alpha} ; q\right)_{\infty} s_{\alpha-1}(z)}{\left(1-a b c d q^{2 \alpha-2}\right)\left(a b q^{\alpha}, a c q^{\alpha}, a d q^{\alpha}, a b c d q^{\alpha-1}, z^{2} ; q\right)_{\infty}}
$$

for $|z|<1$, i.e. $x \in \mathbb{C} /[-1,1]$, and $|a|<1$.

A second OPS satisfying (3.1) but a different initial condition

$$
q_{0}^{\alpha}(x)=1, \quad q_{1}^{\alpha}(x)=1+A_{\alpha}^{-1}\left(z+z^{-1}-a-a^{-1}\right),
$$

which corresponds to the case of zero initial death rate in the birth- and-death model discussed in section 1, was found to be, see [27]:

$$
\begin{aligned}
q_{n}^{\alpha}(x)= & \frac{\left(b c, b d, c d, b c q^{\alpha-1}, b d q^{\alpha-1}, c d q^{\alpha-1}, q^{\alpha}, a z ; q\right)_{\infty} z a^{1-2 \alpha}}{\left(1-a b c d q^{2 \alpha-2}\right)\left(a b q^{\alpha}, a c q^{\alpha}, a d q^{\alpha}, a b c d q^{\alpha-1}, b z, c z, d z, b c d / z ; q\right)_{\infty}} \\
& \times\left\{\left(s_{\alpha-1}(z)-s_{\alpha}(z)\right) r_{n+\alpha}(z)-\left(r_{\alpha-1}(z)-r_{\alpha}(z)\right) s_{n+\alpha}(z)\right\}
\end{aligned}
$$


and hence, for $|z|<1$,

$$
\begin{aligned}
\lim _{n \rightarrow \infty}(z / a)^{n} q_{n}^{\alpha}(x)= & \frac{(a z)^{1-\alpha}\left(b c q^{\alpha-1}, b d q^{\alpha-1}, c d q^{\alpha-1}, q^{\alpha}, a z ; q\right)_{\infty}(a z)^{1-\alpha}}{\left(1-a b c d q^{2 \alpha-2}\right)\left(a b q^{\alpha}, a c q^{\alpha}, a d q^{\alpha}, a b c d q^{\alpha-1}, z^{2} ; q\right)_{\infty}} \\
& \times\left(s_{\alpha-1}(z)-s_{\alpha}(z)\right) .
\end{aligned}
$$

Let us denote the measures of orthogonality for $\left\{p_{n}^{\alpha}(x)\right\}$ and $\left\{q_{n}^{\alpha}(x)\right\}$ by $d \mu^{(1)}(x ; \alpha)$ and $d \mu^{(2)}(x ; \alpha)$, respectively, with

$$
\begin{gathered}
\int_{-\infty}^{\infty} d \mu^{(i)}(x ; \alpha)=1, \quad \int_{-\infty}^{\infty} p_{n}(x) p_{m}(x) d \mu^{(1)}(x ; \alpha)=\pi_{n}^{(1)} \delta_{m n}, \\
\int_{-\infty}^{\infty} q_{n}(x) q_{m}(x) d \mu^{(2)}(x ; \alpha)=\pi_{n}^{(2)} \delta_{m, n},
\end{gathered}
$$

$i=1,2$.

Since $A_{n+\alpha}, B_{n+\alpha}$ and $C_{n+\alpha}$ of (3.1) and (2.14) are bounded if $|a|,|b|,|c|,|d|<1$ and $\alpha \geq 0$ (we shall assume these restrictions to hold) the supports of both $d \mu^{(i)}(t ; \alpha)$, $i=1,2$, are bounded, by [9, Th. IV 2.2] and consequently the moment problem is determined. Since the numerator polynomial corresponding to both $p_{n}^{\alpha}(x)$ and $q_{n}^{\alpha}(x)$ is a multiple of $p_{n-1}^{\alpha+1}(x)$ (which can be easily proved) formula (2.4) now takes the form

$$
\begin{aligned}
\int_{-\infty}^{\infty} \frac{d \mu^{(1)}(t ; \alpha)}{x-t}= & \frac{2 z\left(1-b c d z q^{2 \alpha-1}\right)\left(1-b c d z q^{2 \alpha}\right)}{\left(1-b z q^{\alpha}\right)\left(1-c z q^{\alpha}\right)\left(1-d z q^{\alpha}\right)\left(1-b c d z q^{\alpha-1}\right)} \\
& \times \frac{{ }_{8} W_{7}\left(b c d z q^{2 \alpha} ; b c q^{\alpha}, b d q^{\alpha}, c d q^{\alpha}, q^{\alpha+1}, z q / a ; q, a z\right)}{{ }_{8} W_{7}\left(b c d z q^{2 \alpha-2} ; b c q^{\alpha-1}, b d q^{\alpha-1}, c d q^{\alpha-1}, q^{\alpha}, z q / a ; q, a z\right)}
\end{aligned}
$$

and

$$
\begin{aligned}
\int_{-\infty}^{\infty} \frac{d \mu^{(2)}(t ; \alpha)}{x-t}= & \frac{2 z\left(1-b c d z q^{2 \alpha-1}\right)\left(1-b c d z q^{2 \alpha}\right)}{\left(1-b z q^{\alpha}\right)\left(1-c z q^{\alpha}\right)\left(1-d z q^{\alpha}\right)\left(1-b c d z q^{\alpha-1}\right)} \\
& \times \frac{{ }_{8} W_{7}\left(b c d z q^{2 \alpha} ; b c q^{\alpha}, b d q^{\alpha}, c d q^{\alpha}, q^{\alpha+1}, z q / a ; q, a z\right)}{{ }_{8} W_{7}\left(b c d z q^{2 \alpha-2} ; b c q^{\alpha-1}, b d q^{\alpha-1}, c d q^{\alpha-1}, q^{\alpha}, z / a ; q, a q z\right)}
\end{aligned}
$$

for $x \in \mathbb{C} /[-1,1]$. Using a very useful theorem of Nevai [35, Corollary 36, p. 141, Theorem 40, p. 143] one can show that the support of the absolutely continuous part of the measure is $(-1,1)$ and that the jump function $j(x)$ for the discrete part $d j(x)$ is constant in $(-1,1)$. Furthermore it was shown in [27] that there are no discrete masses outside $(-1,1)$ or at the points $x= \pm 1$. So from $(2.3)$ one deduces that

$$
\begin{aligned}
& \frac{d \mu^{(1)}(\cos \theta ; \alpha)}{d \theta} \\
& \quad=\frac{\left(a b q^{\alpha}, a c q^{\alpha}, a d q^{\alpha}, b c q^{\alpha}, b d q^{\alpha}, c d q^{\alpha}, q^{\alpha+1} ; q\right)_{\infty}}{2 \pi\left(1-a b c d q^{\alpha-2}\right)\left(a b c d q^{\alpha-2}, a b c d q^{2 \alpha} ; q\right)_{\infty}}\left(1-a b c d q^{2 \alpha-2}\right)
\end{aligned}
$$




$$
\begin{aligned}
& \times\left(a b c d q^{2 \alpha-2} ; q\right)_{\infty}\left|\frac{\left(e^{2 i \theta}, q^{\alpha+1} e^{e i \theta} ; q\right)_{\infty}}{\left(a q^{\alpha} e^{i \theta}, b q^{\alpha} e^{i \theta}, c q^{\alpha} e^{i \theta}, d q^{\alpha} e^{i \theta}, q e^{2 i \theta} ; q\right)_{\infty}}\right|^{2} \\
& \times\left|{ }_{8} W_{7}\left(q^{\alpha} e^{2 i \theta} ; q e^{i \theta} / a, q e^{i \theta} / b, q e^{i \theta} / c, q e^{i \theta} / d, q^{\alpha} ; q, a b c d q^{\alpha-2}\right)\right|^{-2} .
\end{aligned}
$$

It was also shown in [27] that $d \mu^{(2)}(x ; \alpha)$ has at most one discrete mass for $0<$ $q<1, \alpha \geq 0$ and $-1<a, b, c, d<1$, and that

$$
\begin{aligned}
& \frac{d \mu^{(2)}(\cos \theta ; \alpha)}{d \theta} \\
& =\frac{\left(a b q^{\alpha}, a c q^{\alpha}, a d q^{\alpha}, b c q^{\alpha}, b d q^{\alpha}, c d q^{\alpha}, q^{\alpha+1} ; q\right)_{\infty}}{2 \pi\left(a b c d q^{2 \alpha} ; q\right)_{\infty}} \\
& \quad \times \frac{\left(a b c d q^{2 \alpha-1} ; q\right)_{\infty}}{\left(a b c d q^{\alpha-1} ; q\right)_{\infty}} \frac{1-2 a x q^{\alpha}+a^{2} q^{2 \alpha}}{1-2 a x+a^{2}} \\
& \quad \times \frac{\left|\left(e^{2 i \theta}, q^{\alpha+1} e^{2 e i \theta} ; q\right)_{\infty}\right|^{2}}{\left|\left(a q^{\alpha} e^{i \theta}, b q^{\alpha} e^{i \theta}, c q^{\alpha} e^{i \theta}, d q^{\alpha} e^{i \theta}, q e^{2 i \theta} ; q\right)_{\infty}\right|^{2}} \\
& \quad \times\left|{ }_{8} W_{7}\left(q^{\alpha} e^{2 i \theta} ; e^{i \theta} / a, q e^{i \theta} / b, q e^{i \theta} / c, q e^{i \theta} / d, q^{\alpha} ; q, a b c d q^{\alpha-1}\right)\right|^{-2} .
\end{aligned}
$$

Explicit forms of $p_{n}^{\alpha}(x)$ and $q_{n}^{\alpha}(x)$ found in [27] are:

$$
\begin{aligned}
& p_{n}^{\alpha}(x)= \sum_{k=0}^{n} \frac{\left(q^{-n}, a b c d q^{2 \alpha+n-1}, a b c d q^{2 \alpha-1}, a e^{i \theta}, a e^{-i \theta} ; q\right)_{k}}{\left(q, a b q^{\alpha}, a c q^{\alpha}, a d q^{\alpha}, a b c d q^{\alpha-1} ; q\right)_{k}} q^{k} \\
& \times{ }_{10} W_{9}\left(a b c d q^{2 \alpha+k-2} ; q^{\alpha}, b c q^{\alpha-1}, b d q^{\alpha-1}, c d q^{\alpha-1}, q^{k+1},\right. \\
&\left.a b c d q^{2 \alpha+n+k-1}, q^{k-n} ; q, a^{2}\right)
\end{aligned}
$$

and

$$
\begin{aligned}
& q_{n}^{\alpha}(x)= \sum_{k=0}^{n} \frac{\left(q^{-n}, a b c d q^{2 \alpha+n-1}, a b c d q^{2 \alpha-1}, a e^{i \theta}, a e^{-i \theta} ; q\right)_{k}}{\left(q, a b q^{\alpha}, a c q^{\alpha}, a d q^{\alpha}, a b c d q^{\alpha-1} ; q\right)_{k}} q^{k} \\
& \times{ }_{10} W_{9}\left(a b c d q^{2 \alpha+k-2} ; q^{\alpha}, b c q^{\alpha-1}, b d q^{\alpha-1}, c d q^{\alpha-1}, q^{k},\right. \\
&\left.a b c d q^{2 \alpha+n+k-1}, q^{k-n} ; q, q a^{2}\right) .
\end{aligned}
$$

Masson's exceptional Askey-Wilson polynomials, see [32], [25] that correspond to the indeterminate cases $a b c d=q$ or $q^{2}$ (see eqn. (2.14)) turn out to be the limiting case $\alpha \rightarrow 0^{+}$of $p_{n}^{\alpha}(x)$, while $q_{n}^{\alpha}(x)$ approaches the Askey-Wilson polynomials.

By using the transformation theory of basic hypergeometric series a simpler form of (3.14) was found in [42] 


$$
\begin{aligned}
p_{n}^{\alpha}(x) \equiv & p_{n}^{\alpha}(x ; a, b, c, d \mid q) \\
= & \frac{\left(a b c d q^{2 \alpha-1}, q^{\alpha+1} ; q\right)_{n}}{\left(q, a b c d q^{\alpha-1} ; q\right)_{n}} q^{-\alpha n} \sum_{k=0}^{n} \frac{\left(q^{-n}, a b c d q^{2 \alpha+n-1} ; q\right)_{k}}{\left(q^{\alpha+1}, a b q^{\alpha} ; q\right)_{k}} \\
& \times \frac{\left(a q^{\alpha} e^{i \theta}, a q^{\alpha} e^{-i \theta} ; q\right)_{k}}{\left(a c q^{\alpha}, a c q^{\alpha} ; q\right)_{k}} q^{k} \sum_{j=0}^{k} \frac{\left(q^{\alpha}, a b q^{\alpha-1}, a c q^{\alpha-1}, a d q^{\alpha-1} ; q\right)_{j}}{\left(q, a b c d q^{2 \alpha-2}, a q^{\alpha} e^{i \theta}, a q^{\alpha} e^{-i \theta} ; q\right)_{j}} q^{j}
\end{aligned}
$$

Assuming that $\max (|a|,|b|,|c|,|d|)<q^{(1-\alpha) / 2}, \alpha>0$, one can derive the following integral representation [42]:

$$
\begin{aligned}
& p_{n}^{\alpha}(x ; a, b, c, d \mid q) \\
= & \int_{-1}^{1} K(x, y) \frac{\left(a b c d q^{2 \alpha-1}, q^{\alpha+1} ; q\right)_{n}}{\left(q, a b c d q^{\alpha-1} ; q\right)_{n}} q^{-\alpha n} p_{n}\left(y ; a q^{\alpha / 2}, b q^{\alpha / 2}, c q^{\alpha / 2}, d q^{\alpha / 2} \mid q\right) d y
\end{aligned}
$$

where

$$
\begin{aligned}
K(x, y)= & \frac{\left(q, q, q, a b q^{\alpha-1}, a c q^{\alpha-1}, a d q^{\alpha-1}, b c q^{\alpha-1}, b d q^{\alpha-1}, c d q^{\alpha-1}, q^{\alpha} ; q\right)_{\infty}}{4 \pi^{2}\left(a b c d q^{2 \alpha-2}, q^{\alpha+1} ; q\right)_{\infty} \sqrt{1-y^{2}}} \\
& \times \frac{\left(e^{2 i \phi}, e^{-2 i \phi} ; q\right)_{\infty}}{h\left(y ; q^{\alpha / 2} e^{i \theta}, q^{\alpha / 2} e^{-i \theta}\right)} \int_{0}^{\pi} \frac{\left(e^{2 i \psi}, e^{-2 i \psi} ; q\right)_{\infty}}{h\left(\cos \psi ; a q^{\frac{\alpha-1}{2}}, b q^{\frac{\alpha-1}{2}}, c q^{\frac{\alpha-1}{2}}, d q^{\frac{\alpha-1}{2}}\right)} \\
& \times \frac{h\left(\cos \psi ; q^{\frac{\alpha+1}{2}} e^{i \theta}, q^{\frac{\alpha+1}{2}} e^{-i \theta}\right.}{h\left(\cos \psi ; q^{1 / 2} e^{i \phi}, q^{1 / 2} e^{-i \theta}\right)} d \psi
\end{aligned}
$$

where $y=\cos \phi, 0 \leq \phi \leq \pi$, and

$$
\begin{aligned}
& h\left(\cos \theta ; a_{1}, a_{2}, \ldots, a_{k}\right):=\prod_{j=1}^{k} h\left(\cos \theta ; a_{j}\right) \\
& h(\cos \theta ; a)=\left(a e^{i \theta}, a e^{-i \theta} ; q\right)_{\infty}=\prod_{k=0}^{\infty}\left(1-2 a q^{k} \cos \theta+a^{2} q^{2 k}\right) .
\end{aligned}
$$

This representation enables us to compute many formulas for the associated polynomials $p_{n}^{\alpha}(x ; a, b, c, d \mid q)$ from the corresponding formulas for the ordinary Askey-Wilson polynomials $p_{n}\left(x ; a q^{\alpha / 2}, b q^{\alpha / 2}, c q^{\alpha / 2}, d q^{\alpha / 2} \mid q\right)$, see, for example, [43].

By replacing $a, b, c, d$ and $\alpha$ in $(3.17)$ by $q^{1 / 2}, q^{\alpha+1 / 2},-q^{\beta+1 / 2},-q^{1 / 2}$ and $c$, respectively, then taking the limit $q \rightarrow 1$ and defining

$$
\begin{aligned}
& P_{n}^{(\alpha, \beta)}(x ; c) \\
= & \frac{(\alpha+c+1)_{n}(\alpha+\beta+c+1)_{n}}{(c+1)_{n}(\alpha+\beta+2 c+1)_{n}} \lim _{q \rightarrow 1} p_{n}^{c}\left(x ; q^{1 / 2}, q^{\alpha+1 / 2},-q^{\beta+1 / 2},-q^{1 / 2} \mid q\right),
\end{aligned}
$$


one can show that

$$
\begin{aligned}
P_{n}^{(\alpha, \beta)}(x ; c)= & \frac{(\alpha+c+1)_{n}}{n !} \sum_{k=0}^{n} \frac{(-n, \alpha+\beta+2 c+n+1)_{k}}{(c+1, \alpha+c+1)_{k}}\left(\frac{1-x}{2}\right)^{k} \\
& \times \sum_{j=0}^{k} \frac{(-k, c, \alpha+c)_{j}}{j !(-k, \alpha+\beta+2 c)_{j}}\left(\frac{2}{1-x}\right)^{j} \\
= & \frac{c \Gamma(\alpha+\beta+2 c)}{\Gamma(\alpha+c) \Gamma(\beta+c)} 2^{1-\alpha-\beta-3 c} \\
& \times \int_{-1}^{1} \int_{-1}^{1} d y d z(1-y)^{\alpha+c-1}(1+y)^{\beta+c-1}(1-z)^{c-1} \\
& \times P_{n}^{(\alpha+c, \beta+c)}\left(\frac{x(1+z)+y(1-z)}{2}\right)
\end{aligned}
$$

$\operatorname{Re}(\alpha+c)>0, \operatorname{Re}(\beta+c)>0, \operatorname{Re} c>0$.

\section{Special and Limiting cases}

I. Continuous $q$-Jacobi polynomials. Let us take

$$
a=q^{1 / 2}, \quad b=q^{\beta+1 / 2}, \quad c=-q^{\alpha+1 / 2}, \quad d=-q^{1 / 2}
$$

and replace $\alpha$ by $c$ in (3.14). The ${ }_{10} \phi_{9}$ then is a balanced, terminating and very-wellpoised series which can be transformed by [14, III. 28]. Thus

$$
\begin{aligned}
& \text { (4.2) }{ }_{10} W_{9}\left(q^{\alpha+\beta+2 c+k} ; q^{c},-q^{\alpha+\beta+c},-q^{\beta+c}, q^{\alpha+c}, q^{k+1}, q^{\alpha+\beta+2 c+n+k+1}, q^{k-n} ; q, q\right) \\
& =\frac{\left(q^{c+1}, q^{\alpha+\beta+2 c+1},-q,-q^{\alpha+\beta+c+1} ; q\right)_{n}\left(q^{\alpha+\beta+c+1},-q^{c+1}, q,-q^{\alpha+\beta+2 c+1} ; q\right)_{k}}{\left(-q^{c+1},-q^{\alpha+\beta+2 c+1}, q, q^{\alpha+\beta+c+1} ; q\right)_{n}\left(-q^{\alpha+\beta+c+1}, q^{c+1},-q, q^{\alpha+\beta+2 c+1} ; q\right)_{k}} \\
& \quad \times{ }_{10} W_{9}\left(-q^{\alpha+\beta+2 c+k} ; q^{c},-q^{\alpha+\beta+c}, q^{\beta+c},-q^{\alpha+c},-q^{k+1}, q^{\alpha+\beta+2 c+n+k+1}, q^{k-n} ; q, q\right) .
\end{aligned}
$$

Hence the explicit form of the associated continuous $q$-Jacobi polynomials is:

$$
\begin{aligned}
& p_{n}^{c}\left(x ; q^{1 / 2}, q^{\beta+1 / 2},-q^{\alpha+1 / 2},-q^{1 / 2} \mid q\right) \\
&= \frac{\left(q^{c+1}, q^{\alpha+\beta+2 c+1},-q,-q^{\alpha+\beta+c+1} ; q\right)_{n}}{\left(q, q^{\alpha+\beta+c+1},-q^{c+1},-q^{\alpha+\beta+2 c+1} ; q\right)_{n}} \sum_{k=0}^{n} \frac{\left(q^{-n}, q^{\alpha+\beta+2 c+n+1},-q^{\alpha+\beta+2 c+1} ; q\right)_{k}}{\left(q^{c+1}, q^{\beta+c+1},-q^{\alpha+c+1} ; q\right)_{k}} \\
& \times \frac{\left(q^{1 / 2} e^{i \theta}, q^{1 / 2} e^{-i \theta} ; q\right)_{k}}{\left(-q,-q^{\alpha+\beta+c+1} ; q\right)_{k}} q^{k} \\
& \times{ }_{10} W_{9}\left(-q^{\alpha+\beta+2 c+k} ; q^{c},-q^{\alpha+\beta+c}, q^{\beta+c},-q^{\alpha+c},-q^{k+1},\right. \\
&\left.q^{\alpha+\beta+2 c+n+k+1}, q^{k-n} ; q, q\right)
\end{aligned}
$$


As $q \rightarrow 1$ this approaches a multiple of Wimp's [49, eqn. (19)] expression for the associated Jacobi polynomials:

$$
\begin{array}{r}
\frac{(c+1)_{n}(\alpha+\beta+2 c+1)_{n}}{n !(\alpha+\beta+c+1)_{n}} \sum_{k=0}^{n} \frac{(-n)_{k}(\alpha+\beta+2 c+n+1)_{k}}{(c+1)_{k}(\beta+c+1)_{k}}\left(\frac{1-x}{2}\right)^{k} \\
\times{ }_{4} F_{3}\left[\begin{array}{c}
k-n, \alpha+\beta+2 c+n+k+1, c, \beta+c \\
c+k+1, \beta+c+k+1, \alpha+\beta+2 c
\end{array} ; 1\right] .
\end{array}
$$

II. Continuous $q$-ultraspherical polynomials. Instead of (4.1) let us now set $b=a q^{1 / 2}, c=-a, d=-a q^{1 / 2}$ and denote

$$
p_{n}^{\alpha}\left(x ; a, a q^{1 / 2},-a-a q^{1 / 2} \mid q\right)=\frac{\left(q^{\alpha+1} ; q\right)_{n}}{\left(a^{4} q^{\alpha} ; q\right)_{n}} a^{n} C_{n}^{\alpha}\left(x ; a^{2} \mid q\right)
$$

where $C_{n}^{\alpha}\left(x ; a^{2} \mid q\right)$ is the associated $q$-ultraspherical polynomials which reduces to the $q$-ultraspherical polynomials

$$
C_{n}\left(x ; a^{2} \mid q\right)=\sum_{k=0}^{n} \frac{\left(a^{2} ; q\right)_{k}\left(a^{2} ; q\right)_{n-k}}{(q ; q)_{k}(q ; q)_{n-k}} e^{i(n-2 k) \theta}
$$

when $\alpha=0$.

Use of the transformation formula $[14,(3.4 .7)]$ and some simplifications gives

$$
\begin{aligned}
C_{n}^{\alpha}\left(x ; a^{2} \mid q\right)= & \frac{\left(a^{4} q^{\alpha-1} ; q\right)_{\infty}}{\left(q^{\alpha+1} ; q\right)_{\infty}} \frac{z^{n}}{1-z^{-2}}{ }_{2} \phi_{1}\left[\begin{array}{r}
q / a^{2} z^{2}, q / a^{2} \\
q / z^{2}
\end{array} ; q, a^{4} q^{\alpha-1}\right] \\
& \times{ }_{2} \phi_{1}\left[\begin{array}{r}
q z^{2} / a^{2}, q / a^{2} \\
q z^{2}
\end{array} ; q, a^{4} q^{\alpha+n}\right]+\left(z \leftrightarrow z^{-1}\right),
\end{aligned}
$$

where $z=e^{i \theta}, x=\cos \theta, 0<\theta<\pi$. From this the generating function follows immediately:

$$
\begin{aligned}
& G_{t}^{\alpha}(x ; \beta \mid q)=\sum_{n=0}^{\infty} C_{n}^{\alpha}(x ; \beta \mid q) t^{n} \\
& =\frac{\left(\beta^{2} q^{\alpha-1} ; q\right)_{\infty}}{\left(q^{\alpha+1} ; q\right)_{\infty}(1-z t)\left(1-z^{-2}\right)}{ }_{2} \phi_{1}\left[\begin{array}{r}
q / \beta, q / \beta z^{2} \\
q / z^{2}
\end{array} ; q, \beta^{2} q^{\alpha-1}\right] \\
& \times{ }_{3} \phi_{2}\left[\begin{array}{r}
q / \beta, q z^{2} / \beta, z t \\
q z^{2}, q z t
\end{array} ; q, \beta^{2} q^{\alpha}\right]+\left(z \leftrightarrow z^{-1}\right),
\end{aligned}
$$

$|t|<1$. We can now combine the two terms on the rhs of (4.8) in the following way. First, note that

$$
G_{t}^{\alpha}(x ; \beta \mid q)=\frac{\left(\beta^{2} q^{\alpha-1} ; q\right)_{\infty}}{\left(q^{\alpha+1} ; q\right)_{\infty}\left(1-z^{-2}\right)} \sum_{j=0}^{\infty} \sum_{k=0}^{\infty} \frac{\left(q / \beta, q / \beta z^{2} ; q\right)_{j}}{\left(q, q / z^{2} ; q\right)_{j}}
$$




$$
\times \frac{\left(q / \beta, q z^{2} / \beta ; q\right)_{k}}{\left(q, q z^{2} ; q\right)_{k}}\left(\beta^{2} q^{\alpha}\right)^{j+k} \frac{q^{-j}\left(1-z^{-2} q^{j-k}\right)}{\left(1-z t q^{k}\right)\left(1-t q^{j} / z\right)} .
$$

Setting $k=n-j$ we find, after some simplifications, that

$$
\begin{aligned}
G_{t}^{\alpha}(x ; \beta \mid q)= & \frac{\left(\beta^{2} q^{\alpha-1} ; q\right)_{\infty}}{\left(q^{\alpha+1} ; q\right)_{\infty}(1-z t)(1-t / z)} \sum_{n=0}^{\infty} \frac{\left(q / \beta, q z^{2} / \beta, z t ; q\right)_{n}}{\left(q, z^{2}, q z t ; q\right)}\left(\beta^{2} q^{\alpha-1}\right)^{n} \\
& \times{ }_{8} W_{7}\left(q^{-n} / z^{2} ; q / \beta, q / \beta z^{2}, t / z, q^{-n} / z t, q^{-n} ; q, \beta^{2}\right)
\end{aligned}
$$

However, using [14, III. 16] and then [14, III.15] we get

$$
\begin{aligned}
& { }_{8} W_{7}\left(q^{-n} / z^{2} ; q / \beta, q / \beta z^{2}, t / z, q^{-n} / z t, q^{-n} ; q, \beta^{2}\right) \\
& \quad=\frac{\left(z^{2}, q / \beta^{2}, q z t ; q\right)_{n}}{\left(q z^{2} / \beta, q / \beta, z t ; q\right)_{n}}{ }_{4} \phi_{3}\left[\begin{array}{c}
q^{-n}, \beta z t, \beta t / z, q \\
\left.q z t, q t / z, q^{-n} / \beta^{2} ; q, q\right] .
\end{array}\right.
\end{aligned}
$$

Hence

$$
\begin{aligned}
G_{t}^{\alpha}(x ; \beta q)= & \frac{\left(\beta^{2} q^{\alpha-1} ; q\right)_{\infty}}{\left(q^{\alpha+1} ; q\right)_{\infty}(1-z t)(1-t / z)} \sum_{n=0}^{\infty} \frac{\left(q / \beta^{2} ; q\right)_{n}}{(q ; q)_{n}}\left(\beta^{2} q^{\alpha-1}\right)^{n} \\
& \times{ }_{4} \phi_{3}\left[\begin{array}{c}
q^{-n}, \beta z t, \beta z / z, q \\
q z t, q t / z, q^{-n} / \beta^{2} ; q, q
\end{array}\right] \\
= & \frac{1-q^{\alpha}}{\left(1-2 x t+t^{2}\right)} 3 \phi_{2}\left[\begin{array}{c}
\beta t e^{i \theta}, \beta t e^{-i \theta}, q \\
q t e^{i \theta}, q t e^{-i \theta} ; q, q^{\alpha}
\end{array}\right],
\end{aligned}
$$

which is the same as [8, eqn. (2.8)].

III. Associated Wilson polynomials. Wilson polynomials $P_{n}(x ; a, b, c, d)$ satisfy the 3 -term recurrence relation

$$
\begin{aligned}
& \left(\lambda_{n}+\mu_{n}-x\right) P_{n}(x)=\lambda_{n} P_{n+1}(x)+\mu_{n} P_{n-1}(x), \\
& P_{-1}(x)=0, \quad P_{0}(x)=1, \quad x=a^{2}+t^{2},
\end{aligned}
$$

where

$$
\begin{aligned}
\lambda_{n} & =\frac{(n+a+b)(n+a+c)(n+a+d)(n+s-1)}{(2 n+s-1)(2 n+s)} \\
\mu_{n} & =\frac{n(n+b+c-1)(n+b+d-1)(n+c+d-1)}{(2 n+s-2)(2 n+s-1)} \\
s & =a+b+c+d
\end{aligned}
$$

see [47], [48] and [3]. 
Wilson [47] found that they are orthogonal on $(-\infty, \infty)$ wrt the weight function

$$
\left|\frac{\Gamma(a+i t) \Gamma(b+i t) \Gamma(c+i t) \Gamma(d+i t)}{\Gamma(2 i t)}\right|^{2}
$$

and that

$$
P_{n}(x ; a, b, c, d)={ }_{4} F_{3}\left[\begin{array}{c}
-n, n+s-1, a-i t, a+i t \\
a+b, a+c, a+d
\end{array} ; 1\right] .
$$

The associated Wilson polynomials are, of course, the solutions of (4.13) with $n$ replaced by $n+\alpha$ in $\lambda_{n}$ and $\mu_{n}$. Their weight function and the orthogonality properties were worked out by Ismail et al. [26], see also [33] and [24]. In fact, the authors in [26] found two families of polynomials that correspond to the two cases we have considered in the previous section. Their weight function [26, (3.39)] for the first family can be found as the $q \rightarrow 1^{-}$limit of our formula (3.12) after having replaced $a, b, c, d, \alpha, a b c d$, $e^{i \theta}$ by $q^{a}, q^{b}, q^{c}, q^{d}, q^{\gamma}$ and $q^{i \tau}$, respectively. Similarly, formula [26, (4.25)] follow as the $q \rightarrow 1^{-}$limit of (3.13) with the same replacements. Furthermore, the functional forms for the two polynomial systems are given by

$$
\begin{aligned}
& P_{n}^{\gamma}(x ; a, b, c, d)=\sum_{k=0}^{n} \frac{(-n, s+2 \gamma+n-1, s+2 \gamma-1, a+i \tau, a-i \tau)_{k}}{k !(a+b+\gamma, a+c+\gamma, a+d+\gamma, s+\gamma-1)_{k}} \\
&{ }_{9} F_{8}(s+2 \gamma+k-2 ; \gamma, b+c+\gamma-1, b+d+\gamma-1, \\
&\quad c+d+\gamma-1, k+1, s+2 \gamma+n+k-1, k-n ; 1)
\end{aligned}
$$

as the $q \rightarrow 1^{-}$limit of (3.14), and

$$
\begin{aligned}
& Q_{n}^{\gamma}(x ; a, b, c, d) \\
= & \sum_{k=0}^{n} \frac{(-n, s+2 \gamma+n-1, s+2 \gamma-1, a+i \tau, a-i \tau)_{k}}{k !(a+b+\gamma, a+c+\gamma, a+d+\gamma, s+\gamma-1)_{k}} \\
& \times{ }_{9} F_{8}(s+2 \gamma+k-2 ; \gamma, b+c+\gamma-1, b+d+\gamma-1, c+d+\gamma-1, k, \\
& \quad+2 \gamma+n+k-1, k-n ; 1),
\end{aligned}
$$

as the $q \rightarrow 1^{-}$limit of (3.15), where

$$
\begin{aligned}
&{ }_{9} F_{8}(a ; b, c, d, e, f, g, h,) \\
&:= \sum_{n=0}^{\infty} \frac{a+2 n}{a} \\
& \frac{(a, b, c, d, e, f, g, h)_{n}}{n !(1+a-b, 1+a-c, 1+a-d, 1+a-e, 1+a-f, 1+a-g, 1+a-h)_{n}} .
\end{aligned}
$$

Acknowledgement. I wish to express my thanks and appreciation to Mourad Ismail for his help and suggestions in preparing the final version of this paper. 


\section{References}

[1] G. Andrews and R. Askey, Classical orthogonal polynomials, in "Polynomes Orthogonaux et applications", Lecture Notes in Mathematics, \#1171, SpringerVerlag, 1985, pp. 36-82.

[2] R. Askey and M.E.H. Ismail, Recurrence relations, continued fractions and orthogonal polynomials, Mem. Amer. Math. Soc., \#300, 1984.

[3] R. Askey and J.A. Wilson, Some basic hypergeometric polynomials that generalize Jacobi polynomials, Mem. Amer. Math. Soc. 319 (1985).

[4] R. Askey and J.A. Wilson, A set of orthogonal polynomials that generalize the Racah coefficients or 6-j symbols, SIAM J. Math. Anal., 10(1979), 1008-1016.

[5] R. Askey and J. Wimp, Associated Laguerre and Hermite polynomials, Proc. Roy. Soc. Edn., 96A (1984), 15-37.

[6] P. Barrucand and D. Dickinson, On the associated Legendre polynomials, in Orthogonal Expansions and Their Continual Analogues, D.T. Haimo, ed., Southern Illinois University Press, Edwardsville, (1968), pp. 43-50.

[7] R.H. Boyer, Discrete Bessel functions, J. Math. Anal. Appl., 2(1961), pp. 509524.

[8] J. Bustoz and M.E.H. Ismail, The associated ultraspherical polynomials and their q-analogues, Can. J. Math. 34(1982), 718-736.

[9] T.S. Chihara, An Introduction to Orthogonal Polynomials, (1978), Gordon and Breach, New York, N.Y.

[10] T.S. Chihara, Chain sequences and orthogonal polynomials, Trans. Amer. Math. Soc. 104(1962), 1-16.

[11] R. Courant, K.O. Friedrichs, and H. Lewy, Über die partiellen Differenzengleichungen der Physik, Math. Ann., 100(1928), pp. 32-74.

[12] D.J. Dickinson, On certain polynomials associated with orthogonal polynomials, Boll. Un. Mat. Ital., (3) 13 (1958), 116- 124.

[13] A. Erdélyi et al., eds., Higher Transcendental Functions, Vol II, Bateman Manuscript Project (1953), McGraw-Hill.

[14] Gasper, G. and Rahman, M., The Basic Hypergeometric Series, Cambridge University Press, Cambridge, U.K., 1990. 
[15] Grunbaum,

[16] Grunbaum and Haine,

[17] D.P. Gupta, M.E.H. Ismail, and D.R. Masson, Contiguous relations, basic hypergeometric functions and orthogonal polynomials II: Associated big q-Jacobi polynomials, J. Math. Anal. Appl. 171 (1992), 477-497.

$[18] \ldots, \ldots$, Associated continuous Hahn polynomials, Canad. J. Math., 43 (1991), 1263-1280.

[19] W. Hahn, Über Orthogonalpolynome mit drei Parametern, Deutche. Math. 5 (1940-41), 373-378.

[20] P. Humbert, Sur deux polynomes associés aux polynomes de Legendre, Bull. Soc. Math. de France, XLVI (1918), 120-151.

[21] M.E.H. Ismail, J. Letessier, D.R. Masson and G. Valent, Birth and death processes and orthogonal polynomials, in "Orthogonal Polynomials: Theory and Practice", P. Nevai, ed., Kluwer Academic Publishers, The Netherlands, 1990.

[22] M.E.H. Ismail, J. Letessier and G. Valent, Linear birth and death models and associated Laguerre and Meixner polynomials, J. Approx. Th., 56 (1988), 337348 .

[23] M.E.H. Ismail, J. Letessier and G. Valent, Quadratic birth and death processes and associated continuous dual Hahn polynomials, SIAM J. Math. Anal. 20 (1989), 727-737.

[24] M.E.H. Ismail, J. Letessier, G. Valent and J. Wimp, Some results on associated Wilson polynomials, in Orthogonal Polynomials and Their Applications, C. Brezinski, L. Gori and A. Ronveaux, eds., IMACS Annals on Computing and Applied Mathematics, Vol. 9, (1991), pp. 293-298.

[25] M.E.H. Ismail, J. Letessier, G. Valent and J. Wimp, Two families of associated wilson polynomials, Can. J. Math. 42 (1990), 659-695.

[26] M.E.H. Ismail and D.R. Masson, Two families of orthogonal polynomials related to Jacobi polynomials, Rocky Moun. J. Math., 21 (1991), 359-375.

[27] M.E.H. Ismail and M. Rahman, Associated Askey-Wilson polynomials, Trans. Amer. Math. Soc., 328 (1991), 201-237. 
[28] S. Karlin and J. McGregor, The differential equations of birth-and-death processes, and the Stieltjes moment problem, Trans. Amer. Math. Soc. 85 (1957), $489-546$.

[29] __ and __ Linear growth, birth- and-death processes, J. Math. Mech. (now Indiana Univ. Math. J.) 7 (1958), 643-662.

[30] A.P. Magnus, Associated Askey-Wilson polynomials as Laguerre-Hahn orthogonal polynomials, in Orthogonal Polynomials and Their Applications, Proceedings, M. Afaro et al., eds., Springer Lecture Notes Math. \#1329 (1988), 261-278.

[31] D. Masson, Convergence and analytic continuation for a class of regular $C$ fractions, Canad. Math. Bull., 28 (4) (1985), 411- 421.

[32] _ Wilson polynomials and some continued fractions of Ramanujan, Rocky Moun. J. Math., 21 (1991), 489-499.

[33] _ _ Associated Wilson polynomials, Const. Approx. 7 (1991), 521-534.

[34] J. Meixner, Orthogonale Polynomsysteme mit einem besonderen Gestalt der erzeugenden Funktion, J. Lond. Math. Soc., 9 (1934), 6- 13.

[35] P. Nevai, Orthogonal Polynomials, Mem. Amer. Math. Soc. \#213, Providence, R.I., 1979.

[36] F.W.J. Olver, Asymptotics and Special Functions, Academic press, New York, 1974.

[37] G. Palama, Polinomi più generali di altri classici e dei loro associati e relazioni tra essi funzioini di seconda specie, Riv. Mat. Univ. Parma, 4 (1953), 363-383.

[38] F. Pollaczek, Sur une généralisation des polynômes de Legendre, C. R. Acad. Sci. Paris, 228 (1949), 1363-1365.

[39] _ _ Systèmes des polynômes biorthogonaux que généralisent les polynômes ultrasphériques, C. R. Acad. Sci. Paris, 228 (1949), 1998-2000.

[40] __ Sur une famille de polynômes orthogonaux qui contient les polynômes d'Hermite et de Laguerre comme cas limites, C. R. Acad. Sci. Paris, 230 (1950), $1563-2256$.

[41] _ _ Sur une famille de polynômes orthogonaux à quatre paramitres, $C . R$. Acad. Sci. Paris, 230 (1950), 2254-2256.

[42] M. Rahman, Some generating functions for the associated Askey-Wilson polynomials, J. Comp. Appl. Math., 68 (1996), 287-296. 
[43] M. Rahman and Q.M. Tariq, Poisson kernel for the associated continuous $q$ ultraspherical polynomials, Meth. Appl. Anal., 4 (1997), 77-90.

[44] J.A. Shohat and J.D. Tamarkin, The Problem of Moments, revised edition, Math. Surveys, Vol. 1, Amer. Math. Soc., Providence, R.I., 1950.

[45] G. Szegö, Orthogonal Polynomials, 4th edn., Amer. Math. Soc. Coll. Publ., Vol. 23, Providence, R.I., 1975.

[46] H.S. Wall and M. Wetzel, Quadratic forms and convergence regions for continued fractions, Duke Math. J., 11 (1944), 89-102.

[47] J. Wilson, Hypergeometric series, recurrence relations and some orthogonal functions, Doctoral Dissertation, University of Wisconsin, Madison, 1978.

[48] __ Some hypergeometric orthogonal polynomials, SIAM J. Math. Anal., 11 (1980), 690-701.

[49] J. Wimp, Explicit formulas for the associated Jacobi polynomials and some applications, Can. J. Math. 39 (1987), 983-1000.

[50] _ _ Some explicit Padé approximants for the function $\Phi^{\prime} / \Phi$ and a related quadrature formula involving Bessel functions, SIAM J. Math. Anal., 16 (1985), 887-895. 